\title{
Topic Study Group No. 07: Popularization of Mathematics
}

\author{
Christian Mercat, Patrick Vennebush, Chris Budd, Carlota Simões \\ and Jens Struckmeier
}

The Popularization of Mathematics Study Group (TSG7) gathered for the first time in ICME diverse people using interesting and inspiring mathematics to motivate both young people and the general public.

It brought together those who popularize mathematics through live performance, exhibits, the media and outreach programs. The group of about forty people, included lively article discussions, poster presentations and demonstrations.

Enthusiastic practitioners stepped back to reflect on the impact of their actions. We first discuss their goals.

- Democratize mathematics

- Set a healthier relationship with mathematics

- Raise performance in math education

- Share math beauty, power and pervasiveness

- Justify taxpayer's money in research and education.

The means of expression are diverse:

1. Art and science (theater, films, visual arts)

2. Fixed, itinerant, and virtual exhibitions for museums, science centers or non-dedicated spaces. Science or mathematical festival or forums

3. Competitions in mathematics and computer science

Co-chairs: Christian Mercat, Patrick Vennebush.

Team members: Chris Budd, Carlota Simões, Jens Struckmeier.

\section{Mercat $(\bowtie)$}

Université Claude Bernard Lyon 1, Lyon, France

e-mail: christian.mercat@math.univ-lyon1.fr

P. Vennebush

Silver Spring, Discovery Education, Washington, USA

e-mail: patrick.vennebush@ verizon.net; patrick@mathjokes4mathyfolks.com

(C) The Author(s) 2017

G. Kaiser (ed.), Proceedings of the 13th International Congress on Mathematical

Education, ICME-13 Monographs, DOI 10.1007/978-3-319-62597-3_34 
4. Mathematical camps

5. Contact with research mathematics and mathematicians

6. Inquiry/research based projects

7. Math circles/math clubs

8. Recreational mathematics

9. New technologies (apps, websites, ...)

10. International exchanges.

It addresses different audiences and target groups tackling unequal access issues, talent, motivation, gender, social, financial or geographical differences, educational opportunities between countries. This TSG resonated very much with the invited lecture of Ricardo Nemirovski about Informal Mathematics. ${ }^{1}$

Twenty nine submissions were accepted, with seven posters, five oral presentations and seventeen articles. Their discussion was split in six main sessions.

A popular medium for popularization is printed material, nowadays usually supported by website archives, or totally online articles:

- Frédéric Gourdeau, from Laval university (Canada), presented Accromath, ${ }^{2}$ a journal of the Institut des Sciences Mathématiques, a consortium of Canadian universities in the Québec region, and CRM, Center for Mathematical Research, founded in 2006, popularizing mathematics, mainly for secondary schoolers, in French.

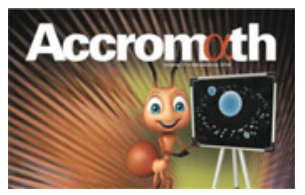

- Vijayakumar Ambat from Cochin university (India), shared his experiences with massive production of math popularization in India, for example through print media with million copies in daily newspapers.

- Nils Berglund introduced us to Images des mathématiques, ${ }^{3}$ a French website, stemming from a previous paper journal, dedicated to current research topics for the general public, written by a large community of professional mathematicians in France.

- The Boletin ${ }^{4}$ is an online journal published since nine years by Juan J. Moreno-Balcázar and his 30+ team in the university of Almería (Spain), that identifies that outreach programs have to go beyond the curriculum in order to show usefulness and beauty of mathematics, rooting on extra motivational aspects such as bilingualism, problem posing and students contests.

\footnotetext{
${ }^{1}$ http://informalmathematics.org/.

${ }^{2}$ http://accromath.uqam.ca/.

${ }^{3}$ http://images.math.cnrs.fr/.

${ }^{4}$ http://boletinmatematico.ual.es.
} 
Shows are another popular way to get a message across while entertaining its audience.

- Andrea Oliveira Hall and Sonia Pais, resp. from the University of Aveiro and Instituto Politécnico de Leira (Portugal), made us laugh and wonder with a mathemagical show, the Mathematical Circus, that since 2012 has pleased more than 10,000 spectators with numerical, topological or combinatorial tricks.

- Nitsa Movshovitz-Hadar, from Technion (Israel), talked about his experience of one-man shows public lectures about mathematics over four years.

Exhibitions, in museums are a traditional form of popularization of knowledge, in many fields but only recently in mathematics.

- Prof. Dr. Albrecht Beutelspacher hold an exhibition excerpt from the Mathematikum ${ }^{5}$ in Gießen (Germany) that since 2002 welcomes hords of visitors, in a formula taken up by the MoMath ${ }^{6}$ in New York.

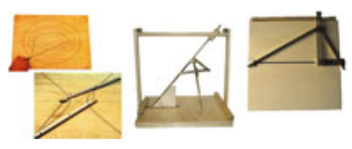

- Michela Maschietto, from the University of Modena (Italy), animates an exhibition of Mathematical Machines that are the content of hands-on activities and trainings, based on historical models and backed up by virtual models. ${ }^{7}$

- The Houses of Mathematics ${ }^{8}$ were presented by Ali Rejali from the University of Isfahan (Iran), as well as similar institutions in Lyon ${ }^{9}$ (France), Quaregnon ${ }^{10}$ (Belgium), Grenoble ${ }^{11}$ (France), Munich ${ }^{12}$ (Germany) or Archimedes Premises in Belgrade (Serbia).

- Abdulkadir Erdoğan from Anadolu University (Turkey) presented (in absentia) examples of popularization activities in the Math School project, an interactive exhibition of workshop games based on Maths à Modeler, ${ }^{13}$ in a specially repurposed classroom.

\footnotetext{
${ }^{5}$ http://www.mathematikum.de/.

${ }^{6} \mathrm{http} / / / \mathrm{momath}$. org/.

${ }^{7}$ http://www.mmlab.unimore.it/.

${ }^{8}$ http://www.mathhouse.org.

${ }^{9}$ http://www.mmi-lyon.fr/.

${ }^{10} \mathrm{https}: / /$ maisondesmaths.be.

${ }^{11} \mathrm{http}: / /$ la-grange-des-maths.fr.

${ }^{12} \mathrm{http}: / / \mathrm{www}-\mathrm{m} 10$.ma.tum.de/ix-quadrat/.

${ }^{13} \mathrm{http}: / /$ mathsamodeler.ujf-grenoble.fr/.
} 
Computers can be used in many different ways to popularize mathematics: as a modern mean of delivery and establishing a community, a way to coordinate collaboration networks and setting virtual laboratories with interactive material. ${ }^{14}$

- Anna Weltman from the University of California, Berkeley (USA) feeds Math Munch, ${ }^{15}$ a popular yet sophisticated math blog targeting middle school students, their teachers and parents. The team of young mathematicians acts as ambassadors of the beauty and fun of math to kids, selecting each month items on the internet and putting them into perspective.

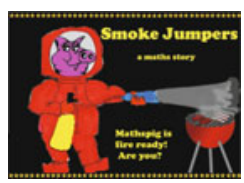

- Kerry Cue from Australia, presented her MathsPigs blog ${ }^{16}$ with lots of down to earth examples of everyday useful mathematics.

- Andreas Daniel Matt and Bianca Violet, from MFO (Germany), invited all contributors to the Imaginary ${ }^{17}$ international project, gathering software, films, posters and virtual models that can be used in order to build hands-on exhibitions around the globe.

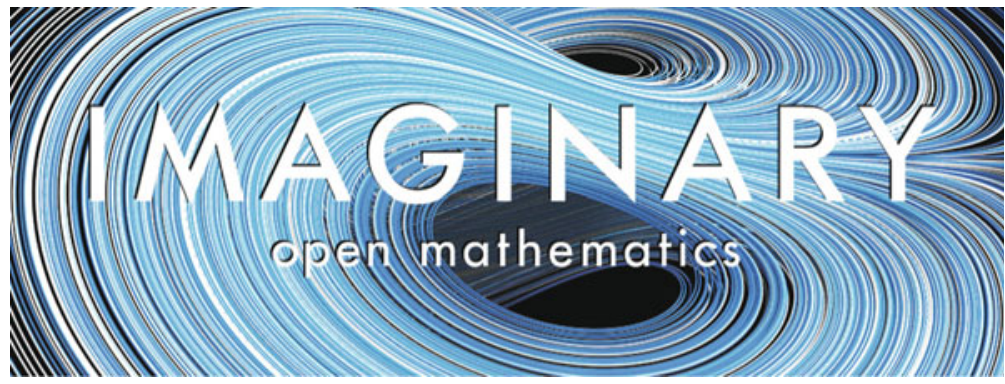

- Ana Cristina Oliveira, from the University of Porto (Portugal), presented Atractor ${ }^{18}$ a Portuguese web based platform of realistic computer drawn simulations of mathematical objects, comprising a youtube channel, including stereo 3D movies, photorealistic virtual interactive exhibits and freeware educational software for elementary school and around the notion of symmetry.

\footnotetext{
${ }^{14} \mathrm{http}: / /$ etudes.ru.

${ }^{15} \mathrm{http}: / /$ www.mathmunch.org/.

${ }^{16} \mathrm{https}: / /$ mathspig.wordpress.com/.

${ }^{17} \mathrm{https}$ ///imaginary.org/.

${ }^{18} \mathrm{http}: / /$ www.atractor.pt/.
} 


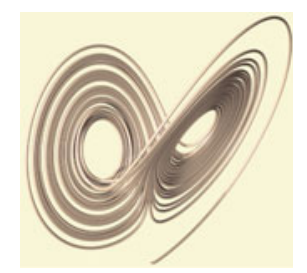

- Sergei Posdniakov from LETI (Russia) introduced computer assisted scientific activities, with virtual laboratories, such as electronic detective, ${ }^{19}$ as a mean to support and motivate more traditional popularization articles.

Handheld mobile devices have their own niche in popularization of mathematics.

- Donna Ann Dietz, from the American University, writes mathematical apps for mobile devices ${ }^{20}$ and evaluates its impact in students' achievements in courses on the content.

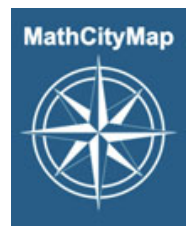

- Adi Nur Cahyono, from Univ. Frankfurt (Germany) and Semarang State Univ. (Indonesia), presented the MathCityMap ${ }^{21}$ project that sets up mathematical modeling tasks that are associated with spots along a city trail and can be explored with a GPS-enabled mobile app.

- Nataly Essonnier from University Claude Bernard Lyon 1 (France), explained how to use Tetrisquiz ${ }^{22}$ and EpsilonWriter in order to gamify easily some math quizz, from simple ones to more sophisticated, usable on a laptop, tablet or handheld device.

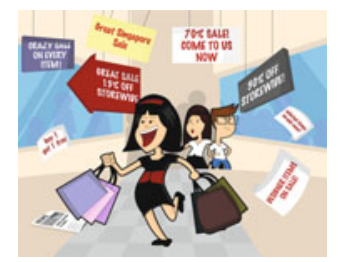

Popular culture, especially comics and games might foster motivation.

\footnotetext{
${ }^{19} \mathrm{http} / / /$ edetective.ipo.spb.ru/.

${ }^{20} \mathrm{http}: / / \mathrm{www} \cdot$ donnadietz.com/.

${ }^{21}$ https://mathcitymap.eu.

${ }^{22} \mathrm{http} / / /$ tquiz.org/.
} 
- Comics storytelling style is used in the Magical ${ }^{23}$ project of the National Institute of Education, Nanyang Tech. Uni. (Singapore), presented by Toh Tin Lam for secondary school students. This non academic contextualization of the material aims at changing the motivation of students.

- Andreas Hinz from LMU Munich (Germany) entertained us with popular puzzling enigmas, requiring ingenuity and patience but as well knowledge, pointing out the unifying force of mathematics, especially graph theory, in their resolution.

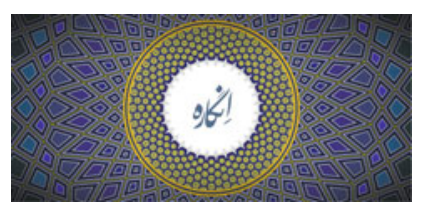

- Mohammad Bahrami from the Shahid Beheshti University, Tehran (Iran), published a poster about the use of interactive magic tricks and islamic art videogame Engare. ${ }^{24}$

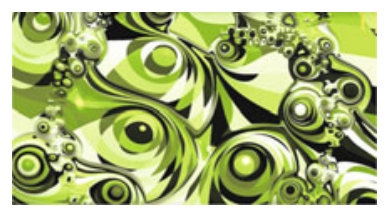

Arts and pictures show the beauty of mathematics.

- Jacinto Eloy Puig Portal from the University of Los Andes (Colombia), presented the incredible artwork of students inspired by mathematics, especially mimicking M.C. Escher ideas using nowadays technology and unleashing the creative potential of mathematical thinking.

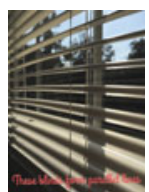

- Emmanuelle Forgeoux from IREM Rennes (France), reported on Mathematical Selfies, ${ }^{25}$ a joint program with Western Carolina University where students illustrate mathematical concepts with photographs and produce inspiring galleries.

Math clubs can be close to the classroom, related to competitions, or during holiday camps.

\footnotetext{
${ }^{23} \mathrm{http}: / /$ math.nie.edu.sg/magical/.

${ }^{24} \mathrm{http} / / /$ www.engare.design/.

${ }^{25} \mathrm{http}: / /$ mathematicalselfies.blogspot.fr/.
} 
- Aviva Szpirglas, from Poitiers University (France) described Math.en.Jeans, ${ }^{26}$ a yearly long initiation to mathematics, with questions introduced by a researcher and followed by a teacher (who is a learner at another level), helping students to go through the frustration and exhilaration of research, until the publication in a national forum, just like professional mathematicians, with poster and lectures in front of hundreds other kids.

- The 180 US Math Circles, ${ }^{27}$ presented by Brandy S. Wieger from Central Washington University (USA), inspired by Eastern European ones, mixes professional mathematicians, K-12 students and their teachers, in a variety of events formats.

Outreach programs can be events, targeting special groups that require special care, mainly in changing the attitude towards mathematics before actually teaching some specific curricular content.

- Violeta Vasilevska, from Utah Valley university (USA), engages, since 2007, women in mathematics and especially, since 2011, high-school girls in the Math Girls $\mathbb{R}$ ock project, ${ }^{28}$ a math club for young women throughout the school year. She presented the project and survey results showing positive attitude shifts, seeing mathematics as more "likable".

- South African senior high school students benefit from a ten day outreach program tapping into adolescents' positive motivation construction strategies, based on self-determination and identity building, driven by autonomy, connectedness and competence, for a lasting effect on students.

- Veronica Sarungi from the Aga Khan University (Tanzania), showcased the Pre-Pi Days events, exhibitions and hands-on activities in lower secondary schools, a fulfilling experience that, in her opinion, ought to be developed in other countries.

Some higher perspectives on popularizing mathematics were taken:

- R. Athmaraman of the Association of Mathematics Teachers of India in Chennai (India), put macro socio-historical and micro perspectives on the attitude towards mathematics. History of ideas, technology and mathematics, as well as psychological studies might inform us of which socio-cultural background can be mathematically friendly, setting an agenda for further research.

- Alix Boissière from Association Plaisir-Maths ${ }^{29}$ (France), introduced the didactical and play-based contract as a tool to assess and design popularization activities.

\footnotetext{
${ }^{26} \mathrm{http}: / /$ www.mathenjeans.fr/.

${ }^{27} \mathrm{http}: / /$ mathcircles.org/.

${ }^{28} \mathrm{https}: / /$ www.uvu.edu/math/mgr/.

${ }^{29} \mathrm{http}$ ://www.plaisir-maths.fr/.
} 
- Martin Andler, from the university of Versailles and president of association Animath $^{30}$ (France), told the lessons learnt in the Cap'Maths project, uniting most popularization activities in mathematics in France in 2012-2106.

- Hong Zhang from the Sichuan University (China), described how historical turmoils make radical changes in math teaching.

Participating in this Topic Study Group has been a very interesting yet challenging experience. We met interesting people, learnt new ways to share our love and passion for mathematics, but the progress towards establishing Popularization of Mathematics as a new respected field of research in math education is yet to come.

Open Access Except where otherwise noted, this chapter is licensed under a Creative Commons Attribution 4.0 International License. To view a copy of this license, visit http://creativecommons. org/licenses/by/4.0/.

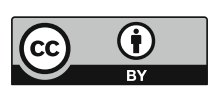

\footnotetext{
${ }^{30} \mathrm{http}: / /$ www.animath.fr/.
} 